\title{
Age group athletes in inline skating: decrease in overall and increase in master athlete participation in the longest inline skating race in Europe - the Inline One-Eleven
}

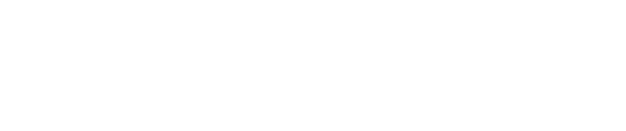

Uwe Teutsch'

Beat Knechtle ${ }^{1,2}$

Christoph Alexander Rüst ${ }^{\prime}$

Thomas Rosemann'

Romuald Lepers ${ }^{3}$

'Institute of General Practice and Health Services Research, University of Zurich, Zurich, Switzerland; ${ }^{2}$ Gesundheitszentrum St Gallen, St Gallen, Switzerland; '3NSERM UI093, Faculty of Sport Sciences, University of Burgundy, Dijon, France
Background: Participation and performance trends in age group athletes have been investigated in endurance and ultraendurance races in swimming, cycling, running, and triathlon, but not in long-distance inline skating. The aim of this study was to investigate trends in participation, age, and performance in the longest inline race in Europe, the Inline One-Eleven over $111 \mathrm{~km}$, held between 1998 and 2009

Methods: The total number, age distribution, age at the time of the competition, and race times of male and female finishers at the Inline One-Eleven were analyzed.

Results: Overall participation increased until 2003 but decreased thereafter. During the 12-year period, the relative participation in skaters younger than 40 years old decreased while relative participation increased for skaters older than 40 years. The mean top ten skating time was $199 \pm 9$ minutes (range: 189-220 minutes) for men and $234 \pm 17$ minutes (range: 211-271 minutes) for women, respectively. The gender difference in performance remained stable at $17 \% \pm 5 \%$ across years.

Conclusion: To summarize, although the participation of master long-distance inline skaters increased, the overall participation decreased across years in the Inline One-Eleven. The race times of the best female and male skaters stabilized across years with a gender difference in performance of $17 \% \pm 5 \%$. Further studies should focus on the participation in the international World Inline Cup races.

Keywords: endurance, men, women, gender

\section{Introduction}

The popularity of endurance sports increased in the last decades. For half-marathons and marathons, the participation has changed over the years. While the overall participation increased for marathons and ultramarathons, ${ }^{1-3}$ the age pattern changed. Jokl et $\mathrm{al}^{4}$ showed that for the New York City Marathon, participation for athletes in the 10-19-year age group decreased, while participation among athletes aged 30-59 years showed a remarkable increase. The finding of an increased participation of master athletes was verified by other authors in marathon running and ultramarathon running. ${ }^{3,5-10}$ Other endurance competitions held in ultraswimming, ${ }^{11-14}$ ultracycling, ${ }^{15-19}$ and ultratriathlons started in the late 20th century. ${ }^{20-22}$

Apart from an increase in master athletes, the increase in female participation was also attributed to an increase in the overall participation in marathon running. ${ }^{4}$ Female participation in endurance and ultraendurance events increased from the early 1980s 
to the present. Jokl et $\mathrm{al}^{4}$ reported that for the New York City Marathon, female participation increased at a greater rate than male participation between 1983 and 1999. Based on the decline in the ratio of male to female runners from 5.6:1 in 1983 to $2.47: 1$ in 1999, and assuming that this pattern would continue, the authors expected the ratio to approach $1: 1$ in 2007. ${ }^{4}$ Participation of female athletes in ultraendurance events exhibited a similar trend. Female participation in ultramarathons such as the Western States 100 Mile Endurance Run progressively increased from $10 \%-12 \%$ of the field in 1986 to $20 \%-22 \%$ since $2001 .^{3}$

It has been assumed that gender differences in performance would vanish for longer running distances in the future. ${ }^{23}$ Although women improved performance at a greater rate than men, there is still a small gap in performance between women and men. Based on the analysis of the world's best running times from 1980 to 1996 for races of $1500 \mathrm{~m}$ and the marathon distance, Sparling et $\mathrm{al}^{24}$ concluded that distance running performances for women had reached a plateau after demonstrating an initial improvement. The comparison of the top 50 running times among women and men showed a gender difference in performance of $10 \%-13 \%$. The reason behind the conclusion that women would outrun men sooner was a comparative analysis of recent or future performances. ${ }^{23,25}$ Coast et al $^{26}$ investigated the gender difference in performance for distances between $100 \mathrm{~m}$ and $200 \mathrm{~km}$ and showed that men were $\sim 12 \%$ faster than women. Longer distances were associated with a greater gender difference in performance. ${ }^{26}$ For the ultramarathon distances, female performance improved relative to men throughout the $1980 \mathrm{~s}$, but in the past two decades, the average times of the fastest runners showed no change for either gender. ${ }^{26}$ The fastest women are still running $~ 20 \%$ slower than the fastest men. ${ }^{27}$

Another key topic addressed in recent years was the investigation of endurance performance related to the age of the athletes. ${ }^{4,5,28,29}$ It was widely accepted that endurance performance showed an age-related decline, as reported for running and for other disciplines such as swimming and triathlon. ${ }^{6,28,30-37}$ Recent research showed that master athletes, defined as athletes older than 35 years,${ }^{38}$ improved their performance times at a higher rate than younger athletes. ${ }^{1,4-6,28}$ Thus, the argument of a decline in endurance performance with advancing age beyond the age of 30-35 years needs to be questioned. ${ }^{39-41}$ The analysis of finishing times of trained endurance athletes showed that the decline in running performance started after the age of 50 years, and that the decline in performance of runners aged beyond 35 years was mostly due to physical inactivity and negative lifestyle factors (eg, smoking, malnutrition, or overnutrition). ${ }^{28}$

Apart from the traditional endurance sports running, ${ }^{1-6}$ cycling, ${ }^{15-18}$ and swimming, ${ }^{11-14}$ new sports disciplines were created in the last two decades, such as long-distance inline skating. ${ }^{42-45}$ In the early 1990 s, the popularity of inline skating showed an enormous increase, leading to inline-speed skating competitions, like sprint races or marathon races such as the Athens-2-Atlanta $(86$ miles $=138.4 \mathrm{~km}) .{ }^{46}$ Inline speed skating is a special endurance discipline because of its technical aspect. Special skates are used where the number, size, and hardness of the wheels can be changed and may affect performance. ${ }^{43}$ According to the race track, the skaters can assemble their skating shoes especially for each road. The use of ordinary skates or custom-made skates, and the different wheel bearings can have a remarkable effect on performance. Apart from anthropometry, training, and prerace experience, equipment is the most important variable for performance of nonexperienced skaters. Performance of experienced skaters who know their equipment relies upon body mass, skin-fold thickness, and body fat. ${ }^{44}$

Recent studies have investigated participation and performance trends for marathon races like the New York City Marathon, ${ }^{1}$ or ultramarathon and long-distance triathlon events like the Ironman Hawaii, ${ }^{20}$ but to date there is no data available on participation and performance trends in inline speed skating races. Studies focused on predictor variables in athletes competing in the long-distance speed skating race, Inline One-Eleven. ${ }^{42,44}$ Knechtle et al $^{42}$ investigated female finishers in the Inline One-Eleven, showing that body height, age, and duration of each training unit were the best predictor variables for female ultraendurance inliners. An investigation focusing on male participants in the Inline One-Eleven demonstrated that the performance of experienced long-distance skaters relied upon body mass, skin-fold thickness, and body fat. ${ }^{44}$

Participation and performance trends in endurance and ultraendurance races have been investigated for different sports disciplines such as swimming, ${ }^{11-14}$ road cycling, ${ }^{15-17}$ mountain bike cycling, ${ }^{18,19}$ running of different distances, ${ }^{2-4,7,9,47-50}$ wheelchair cycling, ${ }^{51,52}$ duathlon, ${ }^{53}$ and triathlon of different race distances $;^{20,22,35-37,54}$ however, these were not investigated for long-distance inline skating. The aim of the present study was to investigate the participation and performance trends of the Inline One-Eleven held in Switzerland from 1998 to 2009. The Inline One-Eleven was the longest inline skating race in Europe, covering the total distance of $111 \mathrm{~km}$ with a total altitude of $1400 \mathrm{~m}$ to climb. The start of the race was in the heart of the city of 
St Gallen, Switzerland, and then went on to a large loop of $111 \mathrm{~km}$ in the east of Switzerland, returning to St Gallen. The race took place each year at the beginning of June. Inline skaters from all over Europe came to St Gallen for the longest inline race in Europe, held on completely closed routes, where drafting was allowed. Due to increased costs, a lack of sponsoring, and a decreased number of participants (only 900 athletes participated in the last race in 2009), the Inline One-Eleven was suspended. According to existing literature, we hypothesized that (1) overall participation in the Inline One-Eleven increased across years; (2) performance of the best athletes improved during the 12-year period of the race; and (3) participation of women and master athletes increased.

\section{Methods}

This study was approved by the institutional review board of St Gallen, Switzerland, with a waiver of the requirement for informed consent given that the study involved the analysis of publicly available data. All race results were provided by the race director. The age at the time of the competition, as well as race times of male and female finishers at the Inline One-Eleven were analyzed from the first race held in 1998 to the last race held in 2009.

\section{Data analysis}

Winner and top ten overall performances

The age at the time of competition and race times of the annual winners and the annual top ten men and women were analyzed from 1998 to 2009. Data (ie, ages and race times) were averaged across the first ten female and male finishers for each year from 1998 to 2009. Race times were converted to minutes. The magnitudes of the gender difference were examined by calculating the percent difference for the race times of the male versus female winners and of the top ten men versus the top ten women.

\section{Age-related changes in performance}

We distinguished between the age group categories of 18-24 years, 25-29 years, 30-34 years, 35-39 years, 40-44 years, 45-49 years, 50-54 years, 55-59 years, 60-64 years, and 65-69 years. Due to the low number of female finishers over 59 years, the last female age group analyzed was 55-59 years. We therefore considered only the performances of the best 50 male and the best 20 female skaters per age group during the 12-year period. This differentiation was selected due to the lower number of women compared to men. For this period, the skating time performances of the best 50 male and the best 20 female skaters per age group was expressed in terms of the percentage of winner performance times.

\section{Statistical analysis}

Data are reported as means \pm standard deviation in the text. Linear regressions were used for estimating the changes of selected variables per year. Pearson's correlation coefficients were used to assess the association between variables (Statistica version 6.1; StatSoft, Inc, Tulsa, OK, USA). The age of the female versus male winners was compared using an unpaired Student's $t$-test. To determine if the age of the top ten male and the female finishers differed across years, an analysis of variance (ANOVA) with repeated measures on year with gender as the between-subject factor was performed. One-way ANOVA was used to compare the race times between the different age groups for both men and women. Tukey's post hoc analyses were used to test differences within the ANOVA when appropriate. Statistical significance was accepted at $P<0.05$.

\section{Results \\ Participation trends}

The Inline One-Eleven started in 1998. In the 12-year period, a total of 8662 finishers could be counted with 1463 female and 7199 male finishers. The number of male finishers indicated a slight increase from 322 to about 500 in the following years until 2002, and then a sharp rise was observed, with a peak of 1026 finishing men in 2003, which was twice as many as in 2002. The number of finishers each year over the history of the event is shown in Figure 1. Between 1998 and 2009, the average number of finishers per year was $600 \pm 190$ (range: 322-1026) for men and $122 \pm 50$ (range: 44-209) for women, respectively. The number of female finishers showed a constant increase from 44 in 1998 to 209 in 2003. Then, the number of finishers showed a constant deterioration again to about 500 men and 100 women finishing the race in 2009. Women accounted on average for $16.5 \% \pm 2.2 \%$ of the field over the 12-year period.

\section{Age of the skaters}

The age distribution of both female and male finishers during the 12-year period is presented in Figure 2. The 5 -year age brackets with the greatest participation were 30-34 years for women and 35-39 years for men. Skaters older than 40 years represented $\sim 41 \%$ of male finishers and $23 \%$ of female finishers. The changes in the percentage of finishers per age group from 1998 to 2009 for both 


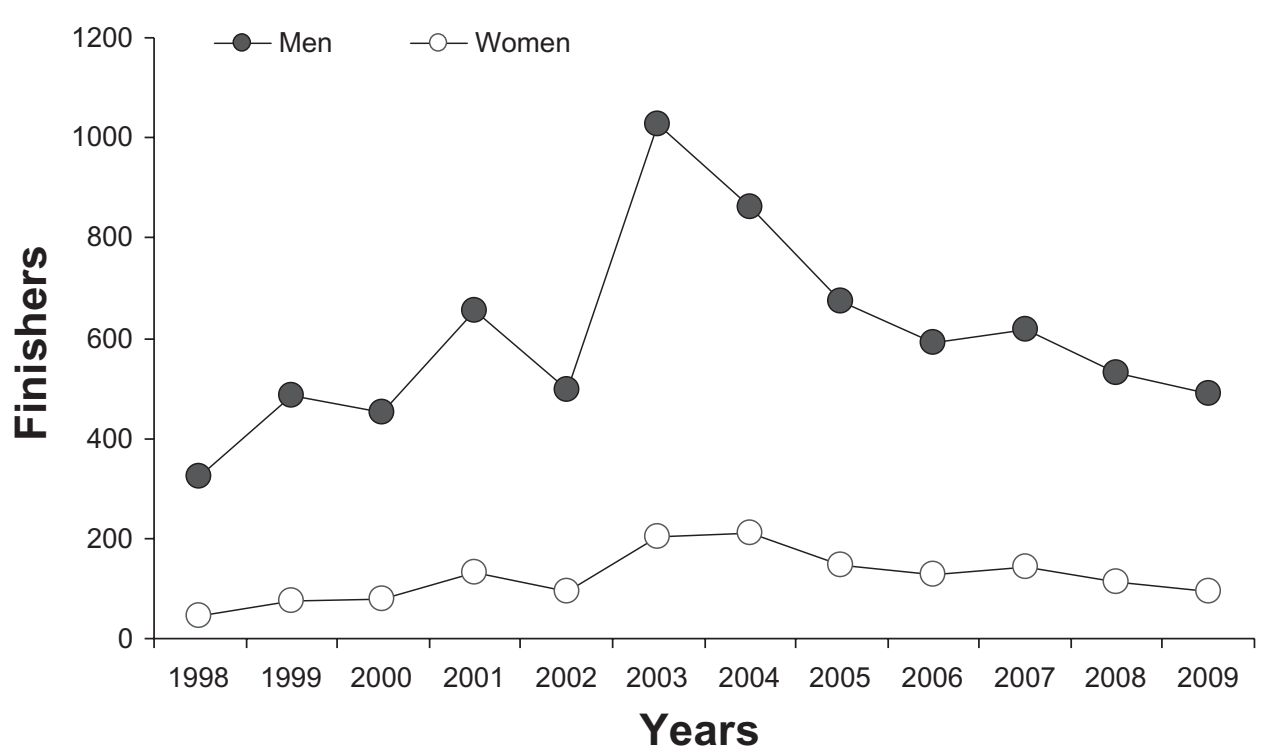

Figure I Number of finishers at the Inline One-Eleven for each gender from 1998 to 2009.

men and women are shown in Figure 3. For both men and women, the percentage of finishers decreased over the 13-year period for age groups 20-29 years (men: $\mathrm{r}=-0.82$, $P=0.0011$; women: $\mathrm{r}=-0.70, P=0.011)$ and $30-39$ years (men: $\mathrm{r}=-0.87, P=0.0002$; women: $\mathrm{r}=-0.53, P=0.075$ ), while it increased for age groups $40-49$ years (men: $\mathrm{r}=0.95, P=0.0001$; women: $\mathrm{r}=0.87, P=0.0002)$ and 50-59 years (men: $\mathrm{r}=0.86, P=0.0003$; women: $\mathrm{r}=0.87$, $P=0.0002)$. The percentage of finishers increased for the men's age group 60-69 years (men: $\mathrm{r}=0.93, P=0.0001$ ), but remained low and stable for the women's age group
60-69 years (women: $\mathrm{r}=-0.57, P=0.06$ ). The ages of both male and female winners are shown in Figure 4A. The ages of the female winners $(25.5 \pm 3.6$ years $)$ were not significantly different compared to male winners (27.5 \pm 3.7 years). Because one-way ANOVA showed no significant difference for the top ten female and male race times between 1998 and 2009, data were pooled together to analyze the age-related changes in skating performances. The mean ages of the top ten placed females and males were $25.8 \pm 2.3$ years and $26.2 \pm 1.8$ years, respectively (Figure 4B).

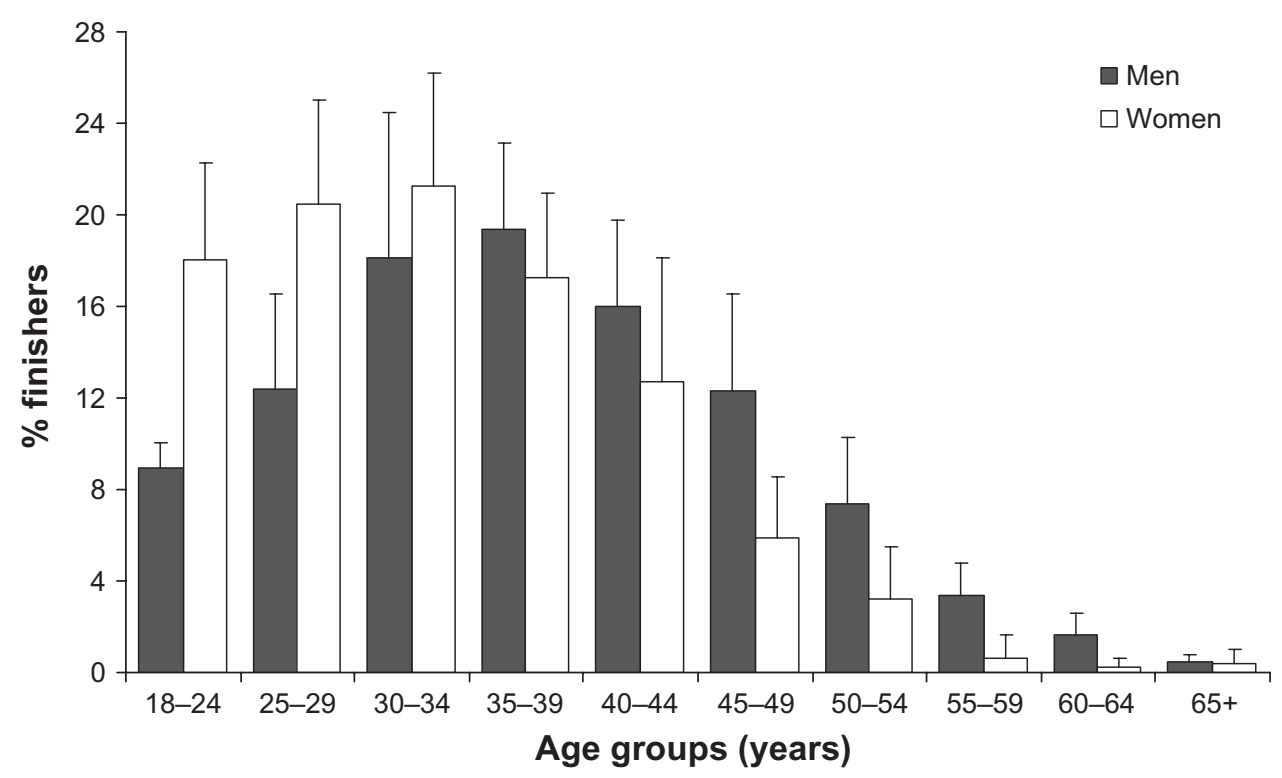

Figure 2 Percentage of female and male finishers per age group over the period 1998-2009. Note: Values are the mean \pm standard deviation. 

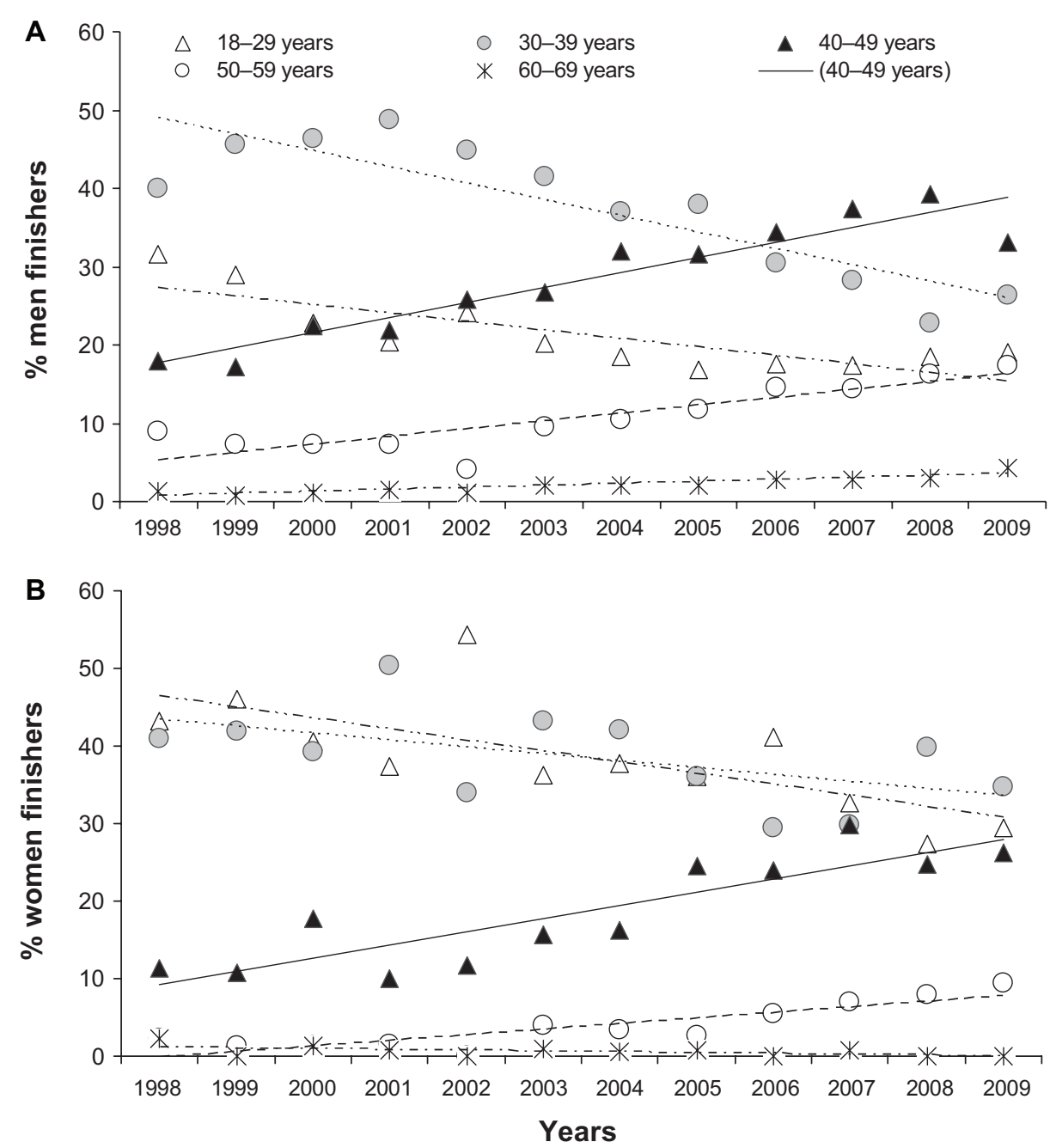

Figure 3 Changes in percentage finishers per age group from 1998 to 2009. (A) Men; (B) Women.

\section{Winner and top ten performance trends}

Figure $5 \mathrm{~A}$ shows the historical performance trends for female and male winners during the studied period. The mean winner race time was $194 \pm 8$ minutes (range: 187-214 minutes) for men and $223 \pm 15$ minutes (range: 208-267 minutes) for women. The average time difference between female and male winners over the 1998-2009 period was equal to $15 \% \pm 5 \%$ (range: 9\%-25\%). Figure 5B shows the historical performance trends of the top ten men and women during the 12-year period. The mean top ten race time was $199 \pm 9$ minutes (range: 189-220 minutes) for men and $234 \pm 17$ minutes (range: 211-271 minutes) for women. The average time difference between the top ten men and women over the 1998-2009 period was equal to $17 \% \pm 5 \%$ (range: $9 \%-24 \%$ ).

\section{Age-related changes in performance}

The mean age-related changes for both female and male race times throughout the 1998-2009 period are presented in Figure 6A. The race times increased in a curvilinear manner with advancing age. There was a significant $(P<0.0001)$ age effect for both female and male race times. No significant difference in time was observed for the three age groups between 18-24 years and 30-34 years for both women and men. For women and men, race time was significantly $(P<0.01)$ greater for the age group 40-44 years and older when compared to the age groups between 18-24 years and 30-34 years. Figure 6B shows the age-related changes in skating performance expressed as a percentage of the winner race time. Compared to the winner, the oldest female age group (55-59 years) was on average 51\% $\pm 18 \%$ slower, while the oldest male age group (65-69 years) was on average $62 \% \pm 20 \%$ slower.

\section{Discussion}

The aims of the present study were to investigate the participation and performance trends of inline skaters competing 

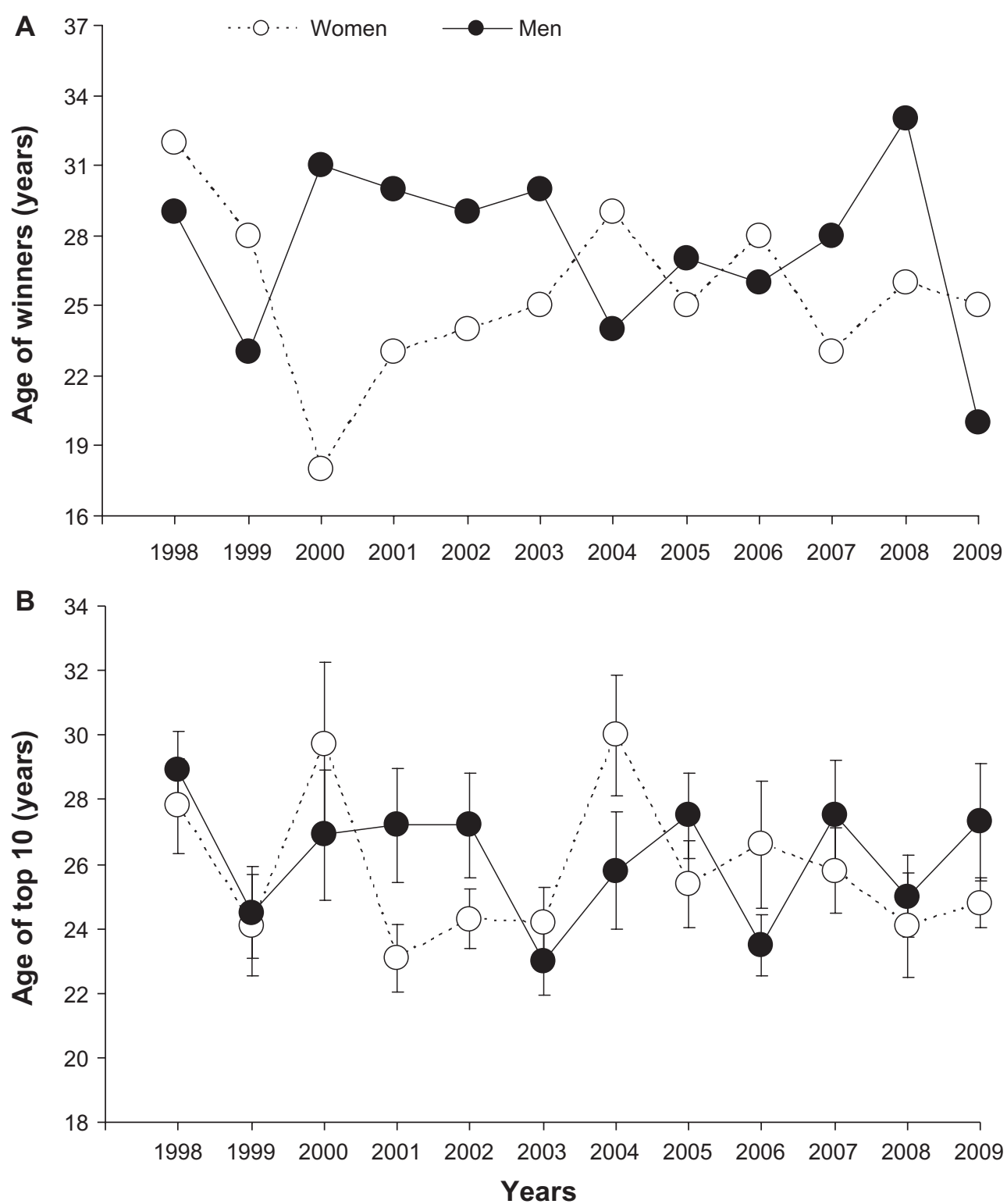

Figure 4 Age of the overall winners and top ten female and male athletes at the Inline One-Eleven from 1998 to 2009 . (A) Age of the female and male winners from 1998 to 2009. (B) Mean ( \pm standard error) age of the overall top ten female and male finishers from 1998 to 2009.

in the Inline One-Eleven held in Switzerland, which was the longest inline skating race in Europe from 1998 to 2009. The main findings were: (1) that after an initial increase from 1998 to 2003 , the overall participation rate in the Inline One-Eleven decreased; (2) the relative participation for skaters older than 40 years increased while it decreased for skaters younger than 40 years over the whole period; and (3) the mean top ten race times remained stable for both men and women.

\section{Participation in Inline One-Eleven}

This is the first study to describe the participation and performance trends of inline skaters in an endurance race such as the Inline One-Eleven. In contrast to our hypothesis, the participation in the race increased only in the beginning years of the race; after 2003, the number of participants diminished until the race was cancelled in 2009. The race was discontinued due to the decreasing number of athletes taking part in the race; other reasons included increasing costs and reduced sponsorship. These findings were in contrast to reports on participation trends in other disciplines like marathon running, ${ }^{4,6}$ ultramarathon running, ${ }^{2,3}$ and the Ironman triathlon, ${ }^{20,55}$ where participation is still growing. One reason for the decreased participation in the Inline OneEleven could be the general diminishing interest in inline skating. ${ }^{56}$ Later, the so-called "trend sport" of inline skating normalized to a public sport and lost its distinctiveness. The 

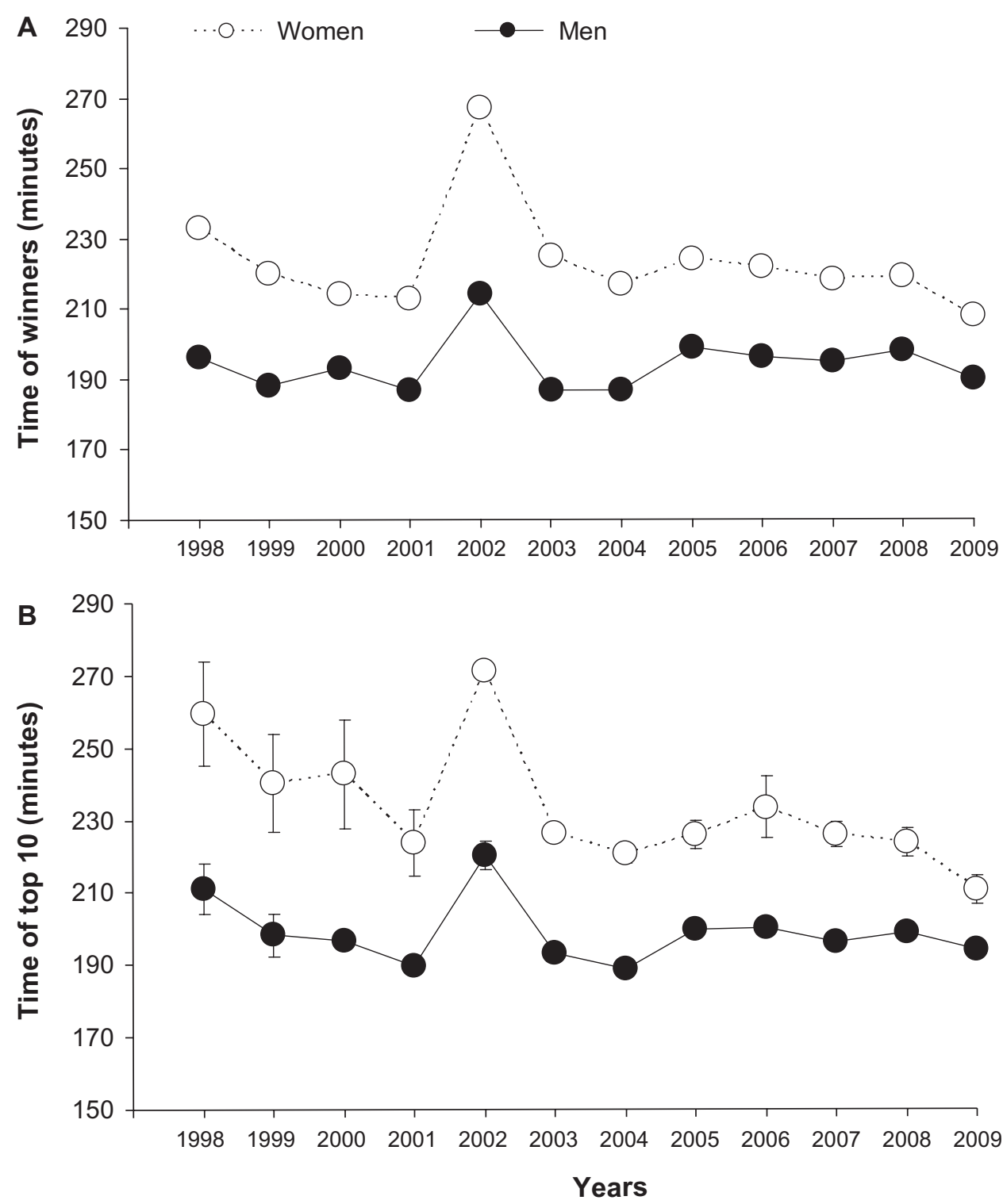

Figure 5 Performance times of the overall and top ten female and male winners at the Inline One-Eleven from 1998 to 2009 . (A) Performance times of the overall female and male winners at the Inline One-Eleven from 1998 to 2009. (B) Mean ( \pm standard deviation) performance times of the overall top ten female and male finishers from 1998 to 2009.

beginning of the 21 st century showed shrinking sales volume and a general diminished interest in inline skating, which may be caused by a lack of acceptable roads where inline skating is allowed. Considered pedestrians, inline-skaters had to use walkways for their sport, which were not adequate at all. In 2009, the Swiss Inline Cup consisted of eight inline events held throughout Switzerland. ${ }^{57}$ Today, a few years later, there are only two of these events left; interestingly, these two events are characterized by a maximum length of $42 \mathrm{~km}$ and a very short lap distance. ${ }^{54}$ There seemed to be a trend towards shorter races, which has also been shown for the Swiss Bike Masters, where the participation showed a dramatic decline since $1998 .{ }^{18,19}$ In addition, there was a tendency of internationalizing the disciplines, leading to more concurrent races worldwide. ${ }^{58,59}$ The resulting effect was that the number of races in every country decreased, such that there existed an acceptable level of participation for at least one or two events every year. Another reason was the fact that inline skating races like the Inline One-Eleven were not considered as ultraendurance events due to their duration of less than 6 hours which, like the Ironman triathlon or the $161 \mathrm{~km}$ marathon, still exhibit increasing participation., ${ }^{2,6}$

This decrease in overall participation might have had a considerable influence on the participation among different genders and age groups. Over the 12-year period studied, the percentage of finishers decreased for athletes in age 

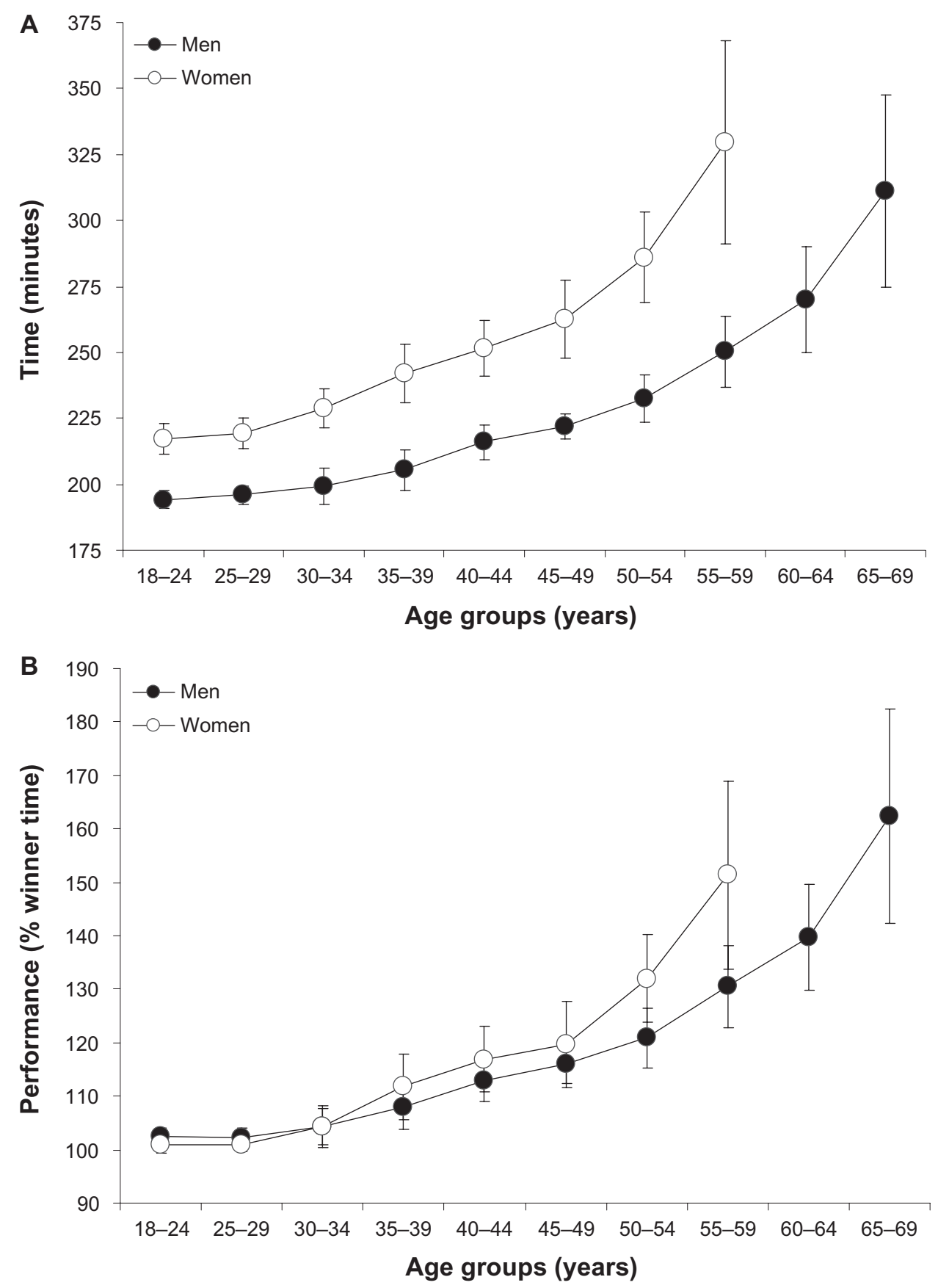

Figure 6 Age-related changes in skating performances at the Inline One-Eleven. (A) Age-related changes in skating performances at the Inline One-Eleven. Data were pooled from 1998 to 2009. Values are means \pm standard deviations. The best 50 male and 20 female skaters per age group were considered during the 12 -year period. For men and women, skating times were significantly greater $(P<0.01)$ for the age group $40-44$ years and older compared to age groups between I8-34 years and 30-34 years. There was no significant difference between the three age groups 18-24 years, 25-29 years, and 30-34 years for both women and men. (B) Age-related changes in skating performances when performance is expressed as a percentage of the winner time.

groups 20-29 years and 30-39 years, while it increased for athletes in age groups 40-49 years and 50-59 years for both men and women. The percentage of finishers slightly increased for men in age group 60-69 years and remained low and stable for women in the age group 60-69 years. These changes in participation might have influenced the statistical analyses.

\section{Age of participants and performances}

A further important finding was that the participation rates for men and women decreased for athletes in the 20-39-year age group, while participation for athletes in the 40-59-year age group showed a remarkable increase. This was expected because similar results were found for other endurance performances like the marathon and half-marathon. ${ }^{4-6}$ 
This could be due to the idea that younger individuals regard endurance sports as leisure activities, and therefore they might quit this type of activity much easier. In contrast, master endurance athletes older than 35 years often practice their endurance sport in order to improve their general physical fitness or to partake in the sport as a health precaution. Older athletes may need more training to achieve their optimal physical condition to take part in an endurance event. Most likely, there must be a greater motivation for athletes to train for an endurance performance event, and therefore the impetus of maintaining the physical status has to be greater. There was a general trend towards an increasing number of healthy aging people in the population being physically able to participate in these events. ${ }^{4}$

The analysis of the age-related performances exposed several findings. The ages of female and male winners were not significantly different with women aged $25.5 \pm 3.6$ years and men aged $27.5 \pm 3.7$ years. The mean age of the top ten women and men across the years remained quite stable at $25.8 \pm 2.3$ and $26.2 \pm 1.8$ years, respectively. Similar age patterns were found in marathon running where the top female and male finishers were in their late $20 \mathrm{~s} .{ }^{60}$ This was in contrast to the results from ultramarathon races like the $100 \mathrm{~km}$ Lauf Biel, where the finishers in the age groups younger than 30 years were slower than finishers in the age groups between 30 and 50 years. ${ }^{50}$ Other studies regarding ultraendurance performance such as the $161 \mathrm{~km}$ marathon also showed faster racing times for older participants aged $30-50$ years. ${ }^{2}$ It seemed that the race times of older participants improved with increasing length of the race..$^{10,48}$

According to Zaryski and Smith, ${ }^{61}$ ultraendurance is defined as competitions lasting at least 6 hours, and successful performance relies on long-term preparation, sufficient nutrition, and accommodation of environmental stressors and psychological toughness. It seemed that these demands were met better by older athletes than by younger ones. The athletes had on average less than 6 hours to complete the race, so it is no ultra-endurance race by definition. This was consistent with the finding that the fastest racers were in the age group 20-29 years, and not in the older groups.

\section{Gender differences in participation and performance}

Regarding participation, female participation was parallel to male participation across years, irrespective of the high rate of male participation in 2003 and 2004, and the decrease in the last years of the race. The rate of female participants was quite stable at $\sim 16 \%$ of all skaters over the 12 -year period. This is in contrast to existing reports about participation in ultraendurance races, where the participation of women and older athletes increased across years. ${ }^{2,3}$ This could be due to the fact that the Inline One-Eleven was not an ultraendurance race.

The highest number of female finishers during the whole 12-year period was observed in the age group 30-34 years, which was quite similar to male participation where the largest group was the age group 35-39 years. Data about the Western States 100 Mile Endurance Run showed the largest participation in the age group 40-44 years for both genders. The percentage of skaters older than 40 years of age was $41 \%$ for men and $23 \%$ for women. Regarding the percentage of finishers per age group, there was no remarkable difference between women and men. Comparing the ages of male and female winners, there was no significant difference between women aged $25.5 \pm 3.6$ years and men aged $27.5 \pm 3.7$ years. Additionally, no difference was apparent in the mean age of the top ten women and top ten men with ages of $25.8 \pm 2.3$ years and $26.2 \pm 1.8$ years, respectively.

We also compared the winners and the top ten performances of women and men. The mean winner race time for men was $194 \pm 8$ minutes and $223 \pm 15$ minutes for women, which means an average time difference between men and women winners of $15 \% \pm 5 \%$ over the studied period. The investigation of the top ten winning times of men and women demonstrated similar results. In running disciplines, females were slower than males and the gender difference increased with increasing race distance. ${ }^{26}$ Comparing the winner times since 2003, the reduction of the gap between the males and females indicates that females showed better improvement than their male counterparts and probably would have outpaced men in the long run.

\section{Limitations}

This cross-sectional data analysis is limited due to the lack of inclusion of training characteristics, ${ }^{44}$ previous experience, ${ }^{45}$ anthropometric characteristics, ${ }^{42}$ aspects of equipment, ${ }^{43}$ and influence of weather and environmental conditions in the analysis. ${ }^{12,53}$ Some athletes might have participated in the race several times across different years, and the inclusion of repeated athletes might have had an influence on the statistical analysis. The nonfinishers were not included due to missing data for nonfinishers in the race results. The inclusion of nonfinishers would give an insight as to whether more women than men were not able to finish the race, and whether more older than younger athletes were among the nonfinishers. ${ }^{62}$ 


\section{Conclusion}

To summarize, overall participation in a long-distance inline skating race such as the Inline One-Eleven decreased, while participation of master athletes increased during the 12-year period. The female participation rate of $16.5 \% \pm 2.2 \%$ and the gender difference in performance of $17 \% \pm 5 \%$ for the annual top ten women and men remained stable. The mean age of the top ten female and male runners was $\sim 26$ years, which was similar to recent findings in marathon and half-marathon distance performances. The race times of the best female and male skaters stabilized across years. The decrease in overall participation was an unexpected finding. Inline skating events with national and international championships are still taking place all over the world. The standard distance for international races is $42 \mathrm{~km}$, with shorter courses increasing in popularity. Further studies should focus on the participation in the international World Inline Cup races. To date, the majority of studies are focusing on injuries related to inline skating. A comparison of participation in nontraditional endurance races such as inline skating and mountain-biking would also be of interest.

\section{Disclosure}

The authors report no conflicts of interest in this work.

\section{References}

1. Burfoot A. The history of the marathon: 1976-present. Sports Med. 2007;37(4-5):284-287.

2. Hoffman MD, Ong JC, Wang G. Historical analysis of participation in $161 \mathrm{~km}$ ultramarathons in North America. Int J Hist Sport. 2010;27(11):1877-1891.

3. Hoffman MD, Wegelin JA. The Western States 100-Mile Endurance Run: participation and performance trends. Med Sci Sports Exerc. 2009;41(12):2191-2198.

4. Jokl P, Sethi PM, Cooper AJ. Master's performance in the New York City Marathon 1983-1999. Br J Sports Med. 2004;38(4):408-412.

5. Leyk D, Erley O, Ridder D, et al. Age-related changes in marathon and half-marathon performances. Int J Sports Med. 2007;28(6): 513-517.

6. Lepers R, Cattagni T. Do older athletes reach limits in their performance during marathon running? Age (Dordr). 2012;34(3):773-781.

7. Zingg MA, Knechtle B, Rüst CA, Rosemann T, Lepers R. Reduced performance difference between sexes in master mountain and city marathon running. Int J Gen Med. 2013;6:267-275.

8. Eichenberger E, Knechtle B, Rüst CA, Rosemann T, Lepers R. Age and gender interactions in mountain ultramarathon running - the Swiss Alpine Marathon. Open Access Journal of Sports Medicine. 2012;3:73-80

9. Zingg M, Rüst CA, Rosemann T, Lepers R, Knechtle B. Master runners dominate the 24-hour ultra-marathons worldwide - a retrospective data analysis from 1998 to 2011. Extreme Physiology and Medicine. In press.

10. Zingg MA, Knechtle B, Rüst CA, Rosemann T, Lepers R. Analysis of participation and performance in athletes by age group in ultramarathons of more than $200 \mathrm{~km}$ in length. Int J Gen Med. 2013;9;6:209-220. doi: 10.2147/IJGM.S43454.
11. Eichenberger E, Knechtle B, Christoph AR, Knechtle P, Lepers R, Rosemann T. No gender difference in peak performance in ultraendurance swimming performance - analysis of the 'Zurich 12-h Swim' from 1996 to 2010. Chin J Physiol. 2012;55(5):346-351.

12. Eichenberger E, Knechtle B, Knechtle P, et al. Sex difference in openwater ultra-swim performance in the longest freshwater lake swim in Europe: sex difference in ultra-swimming. J Strength Cond Res. Epub June 28, 2012

13. Eichenberger E, Knechtle B, Knechtle P, Rüst CA, Rosemann T, Lepers R. Best performances by men and women open-water swimmers during the 'English Channel Swim' from 1900 to 2010. J Sports Sci. 2012;30(12):1295-1301.

14. Fischer G, Knechtle B, Rüst CA, Rosemann T. Male swimmers cross the English Channel faster than female swimmers. Scand J Med Sci Sports. 2013;23(1):e48-e55.

15. Rüst CA, Knechtle B, Rosemann T, Lepers R. Men cross America faster than women - the 'Race Across America' (RAAM) from 1982 to 2012. Int J Sports Physiol Perform. Epub February 20, 2013

16. Zingg M, Knechtle B, Rüst CA, Rosemann T, Lepers R. Age and gender difference in non-drafting ultra-endurance cycling performance the 'Swiss Cycling Marathon'. Extreme Physiology and Medicine. In press.

17. Abou Shoak M, Knechtle B, Knechtle P, Rüst CA, Rosemann T, Lepers R. Participation and performance trends in ultracycling. Open Access Journal of Sports Medicine. 2012;4:41-51.

18. Gloor R, Knechtle B, Knechtle P, et al. Sex-related trends in participation and performance in the 'Swiss Bike Masters' from 1994-2012. Percept Mot Skills. In press.

19. Haupt S, Knechtle B, Knechtle P, Rüst CA, Rosemann T, Lepers R. The age-related performance decline in ultraendurance mountain biking. Res Sports Med. 2013;21(2):146-158.

20. Lepers R. Analysis of Hawaii ironman performances in elite triathletes from 1981 to 2007. Med Sci Sports Exerc. 2008;40(10):1828-1834.

21. Lepers R, Knechtle B, Knechtle P, Rosemann T. Analysis of ultratriathlon performances. Open Access Journal of Sports Medicine. 2011;2011(2):131-136.

22. Knechtle B, Knechtle P, Lepers R. Participation and performance trends in ultra-triathlons from 1985 to 2009. Scand J Med Sci Sports. 2011;21(6):e82-e90.

23. Whipp BJ, Ward SA. Will women soon outrun men? Nature. 1992; 355(6355):25.

24. Sparling PB, O'Donnell EM, Snow TK. The gender difference in distance running performance has plateaued: an analysis of world rankings from 1980 to 1996. Med Sci Sports Exerc. 1998;30(12):1725-1729.

25. Tatem AJ, Guerra CA, Atkinson PM, Hay SI. Athletics: momentous sprint at the 2156 Olympics? Nature. 2004;431(7008):525.

26. Coast JR, Blevins JS, Wilson BA. Do gender differences in running performance disappear with distance? Can J Appl Physiol. 2004;29(2): 139-145.

27. Hoffman MD. Performance trends in $161-\mathrm{km}$ ultramarathons. Int $J$ Sports Med. 2010;31(1):31-37.

28. Leyk D, Erley O, Gorges W, et al. Performance, training and lifestyle parameters of marathon runners aged 20-80 years: results of the PACEstudy. Int J Sports Med. 2009;30(5):360-365.

29. Wright VJ, Perricelli BC. Age-related rates of decline in performance among elite senior athletes. Am J Sports Med. 2008;36(3):443-450.

30. Trappe S. Marathon runners: how do they age? Sports Med. 2007; 37(4-5):302-305.

31. Tanaka H, Seals DR. Endurance exercise performance in Masters athletes: age-associated changes and underlying physiological mechanisms. J Physiol. 2008;586(1):55-63.

32. Lepers R, Sultana F, Bernard T, Hausswirth C, Brisswalter J. Agerelated changes in triathlon performances. Int J Sports Med. 2010; 31(4):251-256.

33. Baker AB, Tang YQ. Aging performance for masters records in athletics, swimming, rowing, cycling, triathlon, and weightlifting. Exp Aging Res. 2010;36(4):453-477. 
34. Bernard T, Sultana F, Lepers R, Hausswirth C, Brisswalter J. Age-related decline in olympic triathlon performance: effect of locomotion mode. Exp Aging Res. 2010;36(1):64-78.

35. Etter F, Knechtle B, Rüst CA, Rosemann T, Lepers R. The age-related decline in Olympic distance triathlon performance differs between males and females. J Sports Med Phys Fitness. In press.

36. Knechtle B, Rüst CA, Knechtle P, Rosemann T, Lepers R. Age-related changes in ultra-triathlon performances. Extreme Physiology and Medicine. 2012;1:5.

37. Etter F, Knechtle B, Bukowski A, Rüst CA, Rosemann T, Lepers R. Age and gender interactions in short distance triathlon performance. J Sports Sci. Epub January 28, 2013.

38. Reaburn P, Dascombe B. Endurance performance in masters athletes. Eur Rev Aging Phys Act. 2008;5:31-42.

39. Fleg JL, Lakatta EG. Role of muscle loss in the age-associated reduction in VO2 max. J Appl Physiol. 1988;65(3):1147-1151.

40. Fuchi T, Iwaoka K, Higuchi M, Kobayashi S. Cardiovascular changes associated with decreased aerobic capacity and aging in long-distance runners. Eur J Appl Physiol Occup Physiol. 1989;58(8):884-889.

41. Hagberg JM. Effect of training on the decline of VO2 max with aging. Fed Proc. 1987;46(5):1830-1833.

42. Knechtle B, Knechtle P, Rosemann T, Lepers R. Is body fat a predictor of race time in female long-distance inline skaters? Asian J Sports Med. 2010;1(3):131-136.

43. Knechtle B, Knechtle P, Rosemann T. A paradigm for identifying ability in competition: the association between anthropometry, training and equipment with race times in male long-distance inline skaters-the 'Inline One Eleven'. Human Movement. 2011;12(2):171-179.

44. Knechtle B, Knechtle P, Rüst CA, Senn O, Rosemann T, Lepers R. Predictor variables of performance in recreational male long-distance inline skaters. J Sports Sci. 2011;29(9):959-966.

45. Knechtle B, Knechtle P, Rüst CA, Rosemann T, Lepers R. Age, training, and previous experience predict race performance in longdistance inline skaters, not anthropometry. Percept Mot Skills. 2012;114(1):141-156.

46. Georgia International Road Skating. Athens to Atlanta Road Skate [homepage on the Internet]. Atlanta, GA: Georgia International Road Skating. Available from: http://www.a2a.net. Accessed March 9, 2013.

47. Knoth C, Knechtle B, Rüst CA, Rosemann T, Lepers R. Participation and performance trends in multistage ultramarathons - the 'Marathon des Sables' 2003-2012. Extreme Physiology and Medicine. 2012;1:13.

48. Da Fonseca-Engelhardt K, Knechtle B, Rüst CA, Knechtle P, Lepers R, Rosemann T. Participation and performance trends in ultra-endurance running races under extreme conditions - 'Spartathlon' versus 'Badwater'. Extreme Physiology and Medicine. In press.
49. Rüst CA, Knechtle B, Rosemann T, Lepers R. Analysis of performance and age of the fastest 100-miles ultra-marathoners worldwide. Clinics (Sao Paulo). In press.

50. Knechtle B, Rüst CA, Rosemann T, Lepers R. Age-related changes in 100-km ultra-marathon running performance. Age (Dordr). 2012;34(4):1033-1045.

51. Lepers R, Stapley PJ, Knechtle B. Gender differences in wheelchair marathon performance - Oita International Wheelchair Marathon from 1983 to 2011. Open Access Journal of Sports Medicine. 2012;2012(3):169-174.

52. Lepers R, Stapley PJ, Knechtle B. Analysis of marathon performances of disabled athletes. Movement and Sport Sciences. In press.

53. Rüst CA, Knechtle B, Knechtle P, et al. Gender difference and agerelated changes in performance at the long distance duathlon. J Strength Cond Res. 2013;27(2):293-301.

54. Knechtle B, Rüst CA, Rosemann T, Lepers R. Age and gender differences in half-Ironman triathlon performances - the Ironman 70.3 Switzerland from 2007 to 2010. Open Access Journal of Sports Medicine. 2012;3:59-66.

55. Lepers R, Maffiuletti NA. Age and gender interactions in ultraendurance performance: insight from the triathlon. Med Sci Sports Exerc. 2011;43(1):134-139.

56. For the sport of rollerblading, it's inline and in decline [webpage on the Internet]. Newark, DE: The Review, University of Delaware; 2011 Available from: http:/www.udreview.com/editorial/for-the-sport-ofrollerblading-it-s-inline-and-in-decline-1.1972265\#.UTr4jjdWKVo. Accessed March 9, 2013.

57. Iguana Marketing AG. Swiss Inline Cup [webpage on the Internet] Dübendorf: Iguana Marketing AG; 2010. Available from: http://www. swiss-inline-cup.ch/. Accessed March 9, 2013.

58. Inline Planet [homepage on the Internet]. Inline Planet. Available from: http://www.inlineplanet.com. Accessed March 12, 2013.

59. Williams C. North American inline skating marathons: find a marathon that is just right for you [webpage on the Internet]. New York, NY: About.com. Available from: http://inlineskating.about.com/ od/competitiveinlineskating/tp/northam_marathons.htm. Accessed March 12, 2013.

60. Hunter SK, Stevens AA, Magennis K, Skelton KW, Fauth M. Is there a sex difference in the age of elite marathon runners? Med Sci Sports Exerc. 2011;43(4):656-664.

61. Zaryski C, Smith DJ. Training principles and issues for ultra-endurance athletes. Curr Sports Med Rep. 2005;4(3):165-170.

62. Knechtle B, Knechtle P, Rüst CA, Rosemann T, Lepers R. Finishers and nonfinishers in the 'Swiss Cycling Marathon' to qualify for the 'Race Across America'. J Strength Cond Res. 2011;25(12):3257-3263.
International Journal of General Medicine

\section{Publish your work in this journal}

The International Journal of General Medicine is an international, peer-reviewed open-access journal that focuses on general and internal medicine, pathogenesis, epidemiology, diagnosis, monitoring and treatment protocols. The journal is characterized by the rapid reporting of reviews, original research and clinical studies across all disease areas.

\section{Dovepress}

A key focus is the elucidation of disease processes and management protocols resulting in improved outcomes for the patient.The manuscript management system is completely online and includes a very quick and fair peer-review system. Visit http://www.dovepress.com/ testimonials.php to read real quotes from published authors. 